\title{
Similaridade do sítio de vocalização em uma comunidade de anfíbios anuros na região noroeste do Estado de São Paulo, Brasil
}

\author{
Denise de C. Rossa-Feres ${ }^{1}$ \\ Jorge $\mathrm{Jim}^{2}$
}

\begin{abstract}
Similarity of call site in anuran community in Northwestern São Paulo State, Brazil (Amphibia, Anura). The breadth and overlap of male call site were determined for 15 anuran species from a temporary pond in Northwestern São Paulo State, Brazil. Four measurements were made at the point of capture of each calling male and the frequency of mating call was determined. There was a high overlap along each variable of call site, but less than $28 \%$ of possible species pairs presented high overlap for all variables. Taxonomically related species pairs calling from grass presented spatial and acoustic segregation, while those calling from the ground presented only acoustic segregation. The similarity of call site seems to be not only related to the taxonomic relationships but also with the body size and spatial heterogeneity.

KEY WORDS. Anura, vocalization site, microhabitat, community ecology, similarity at call site
\end{abstract}

Muitos estudos sobre comunidades de anuros têm constatado que a estrutura física do canto e o sítio de vocalização foram, nessa ordem, os fatores mais importantes na segregação das espécies (DUELLMAN 1967; CREUSERE \& WHITTFORD 1976; CARDOSO et al. 1989; CARDOSO \& VIELLIARD 1990). Estudando comunidades de anuros em região tropical, BOWKER \& BOWKER (1979), CARDOSO et al. (1989), HEYER et al. (1990) e HADDAD \& SAZIMA (1992) registraram segregação espacial do sítio de vocalização entre diferentes espécies.

O enfoque nas espécies congenéricas (OLDHAM \& GERHARDT 1975; BOWKER \& BOWKER 1979; DiAZ \& VALENCIA 1985; HEYER et al. 1990) tem revelado que o grau de sobreposição entre as espécies próximas é variável (HEYER \& BELLIN 1973; BOWKER \& BOWKER 1979; ETGES 1987), mas que, por outro lado, sobreposição total parece rara. HEYER et al. (1990) registraram sobreposição total entre apenas quatro, de 17 pares de espécies próximas estudadas em uma comunidade de anuros de Mata Atlântica. HEYER \& BELLIN (1973) encontraram grandes valores de sobreposição de nicho entre três espécies de Leptodactylus Fitzinger, 1826, mas cada uma pertencente a diferentes grupos de espécies; além disso, duas delas apresentaram segregação espacial do sítio de vocalização. BoWKER \& BoWKER (1979), estudando uma comunidade com dez espécies de anuros concluíram que, embora tenha ocorrido sobrepo-

1) Departamento de Zoologia e Botânica, Universidade Estadual Paulista. 15054-000 São José do Rio Preto, São Paulo, Brasil. E-mail: denise@zoo.ibilce.unesp.br

2) Departamento de Zoologia, Universidade Estadual Paulista. 18618-000, Botucatu, São Paulo, Brasil. 
sição espacial e temporal entre os indivíduos de todas as espécies, o fato de cada uma possuir período e local onde é mais abundante, pode ser importante na redução da probabilidade de cruzamento heteroespecífico.

Analisando a partilha de recursos em uma comunidade com 64 espécies de anuros, HEYER et al. (1990) sugerem que há um padrão nos tipos de mudanças ecológicas que ocorreram entre espécies de morfologia semelhante e que resultaram em partilha de recursos. Segundo os autores, espécies próximas tendem a ter o mesmo tipo de vida, mas em partes do hábitat levemente diferentes, de modo que elas são espacialmente separadas. Para verificar o quão geral é o padrão sugerido por HeYER et al. (1990), no presente estudo determinou-se o sítio de vocalização de 15 espécies de uma comunidade de anuros, das quais oito constituem pares de espécies taxonomicamente próximas.

\section{MATERIAL E MÉTODOS}

O estudo foi desenvolvido em um açude temporário, que acumula água de chuva durante cinco a sete meses por ano, escavado em uma área de pastagem em Nova Itapirema, Distrito de Nova Aliança ( $21^{\circ} 11^{\prime}$ 'S $\left.49^{\circ} 42^{\prime} \mathrm{W}\right)$, Estado de São Paulo. $\mathrm{O}$ clima da região é tropical, com precipitação anual variando entre 1.100 e 1.250 mm, a maior parte concentrada na estação chuvosa, de outubro a março, e apenas 15\% precipitando na estação seca, de abril a setembro (ARID \& BARCHA 1973). O mês de início da estação chuvosa varia a cada ano e as chuvas são inconstantes, não impedindo que o açude seque uma ou duas vezes, após as primeiras chuvas (observação do primeiro autor).

$\mathrm{O}$ açude possui dimensões aproximadas de $54 \times 22 \times 0,7 \mathrm{~m}$ no período de maior volume d'água. O solo é argiloso e cerca da metade de seu perímetro é formado por margens em barranco, com cerca de $1 \mathrm{~m}$ de altura, e o restante por margens planas. A vegetação é constituída por gramíneas marginais e emergentes (Poaceae e Cyperaceae), macrófitas (Nympheaceae e Polygonaceae) e dois pequenos agrupamentos de arbustos (Solanaceae) na parte alta das margens em barranco.

Observações de campo foram desenvolvidas em 41 noites, no período de outubro de 1992 a março de 1995. Essas observações tiveram freqüência mensal na estação seca, quinzenal na estação chuvosa de 1992/1993 e semanal nas estações chuvosas de 1993/1994 e de 1994/1995, sendo iniciadas ao escurecer, entre 18:00 e 19:00 horas, e finalizadas quando a emissão de vocalização pelos anuros diminuía, entre 21:00 e 24:00 horas.

Para a determinação do sítio de vocalização, o açude foi percorrido duas ou três vezes ao longo do seu perímetro, partindo de um ponto inicial diferente a cada noite. $\mathrm{O}$ percurso não foi linear, já que machos adultos podiam estar vocalizando na área central ou externamente às margens do açude. Para cada macho ativo localizado, foram anotadas as seguintes variáveis do sítio de vocalização: a) distância da margem, b) tipo de substrato e c) direcionamento em relação à margem. Além disso, para os anuros que vocalizaram empoleirados foi determinada a altura na vegetação (d), enquanto para os que vocalizaram apoiados sobre o solo ou flutuando na água foi determinada a profundidade da coluna d'água (e). 
Para a análise estatística foram consideradas somente as espécies com número de observações maior ou igual a cinco. A sobreposição de nicho foi calculada separadamente entre os anuros que vocalizaram empoleirados e entre os que vocalizaram apoiados sobre o solo ou flutuando na água, através do índice de similaridade de Morisita-Horn (KREBS 1989), para os dados de freqüência por categoria de cada variável do sítio de vocalização. A sobreposição multidimensional foi calculada considerando todas as variáveis simultaneamente. Para verificar a existência de um padrão no uso de recursos, na matriz obtida com o índice de Morisita-Horn foi aplicada análise de agrupamento, através da técnica de média não ponderada. A amplitude do sítio de vocalização foi calculada através do índice de Levins (HEYER \& BELLIN 1973; KREBS 1989). Além disso, como oito das 15 espécies da comunidade estudada formaram pares de espécies taxonomicamente próximas, a sobreposição de nicho foi discutida tanto no contexto da comunidade de anuros quanto entre as espécies próximas. Foram consideradas taxonomicamente próximas as espécies congenéricas pertencentes ao mesmo grupo intragenérico: Hyla nana Boulenger, 1889 e H. sanborni Schmidt, 1944; Physalaemus cuvieri Fitzinger, 1826 e P. centralis Bokermann, 1962; P. nattereri (Steindachner, 1863) e P. fuscomaculatus (Steindachner, 1864); Scinax fuscovarius (A. Lutz, 1925) e $S$. similis (Cochran, 1952). As demais espécies foram: Bufo paracnemis A. Lutz, 1925, Elachistocleis ovalis (Schneider, 1799), Hyla raniceps (Cope, 1826), Leptodactylus fuscus (Schneider, 1799), L. podicipinus (Cope, 1952), Pseudis paradoxa (Linnaeus, 1758), Scinax fuscomarginatus (A. Lutz, 1925).

Para verificar se as médias das variáveis profundidade, altura e distância da margem diferiram entre os machos de cada um dos quatro pares de espécies taxonomicamente próximas, foi utilizado o teste t de Student (VIEIRA 1985). No texto são utilizadas as abreviações: (X) média, (DP) desvio padrão, (CV) coeficiente de variação, $(\mathrm{gl})$ graus de liberdade, $(\mathrm{t})$ teste $\mathrm{t},(\mathrm{B})$ índice de Levins, $\left(\mathrm{C}_{\mathrm{H}}\right)$ índice de similaridade de Morisita-Horn.

$\mathrm{O}$ registro das vocalizações dos machos adultos foi obtido com gravador Sony TCM-S64V, com microfone cardióide Le Son LLZ, de baixa impedância. As gravações foram feitas à distância de $0,5 \mathrm{~m}$ a $1,5 \mathrm{~m}$ do indivíduo em atividade de vocalização. Uma análise qualitativa da estrutura física do canto de anúncio foi efetuada em computador Macintosh com digitalizador de som e programa MacRecorder Sound System 2.0.5, no Laboratório de Bioacústica, Universidade Estadual Paulista (UNESP), Rio Claro, São Paulo.

\section{RESULTADOS}

Dentre as 18 espécies de anuros registradas no açude temporário, três foram raras, apresentando abundância anual menor que cinco indivíduos: Hyla minuta, Leptodactylus gr. ocellatus e Pseudopaludicola mystacalis. As características do sítio de vocalização foram, então, analisadas para as 15 espécies restantes (Tabs I, II).

A maioria dos machos de Scinax fuscovarius vocalizou sobre o solo, distantes da margem do açude, enquanto os de $S$. similis também vocalizaram distantes da margem, mas empoleirados na vegetação arbustiva (Tab. I). A média de altura do sítio de vocalização de $S$. fuscovarius e de $S$. similis foi significativamente 
diferente $(\mathrm{t}=5,64, \mathrm{gl}=19, \mathrm{p}<0,01)$, enquanto as médias de distância da margem não diferiram. Os machos de $S$. fuscovarius vocalizaram com a cabeça voltada para o centro do açude, enquanto os machos de $S$. similis não apresentaram padrão no seu direcionamento (Tab. I).

Tabela I. Distribuição de freqüência ao longo das cinco variáveis do sítio de vocalização, determinadas para as seis espécies que vocalizaram empoleiradas na vegetação, no açude temporário em Nova Itapirema, São Paulo. (Hn) Hyla nana, (Hr) H. raniceps, (Hs) H. sanborni, (Sfm) Scinax fuscomarginatus, (Sfu) S. fuscovarius, (Ssi) S. similis; os números em negrito, na frente de cada variável, indicam o número de observações para cada espécie.

\begin{tabular}{|c|c|c|c|c|c|c|c|}
\hline \multicolumn{2}{|c|}{ Sítio de vocalização } & $\mathrm{Hn}$ & Hs & Sfu & Ssi & Sfm & $\mathrm{Hr}$ \\
\hline \multicolumn{2}{|c|}{ Altura na vegetaçăo $(\mathrm{cm})$} & 574 & 70 & 28 & 18 & 222 & 5 \\
\hline \multicolumn{2}{|c|}{$0-10$} & 20,2 & 0,0 & 92,8 & 27,8 & 5,0 & 40,0 \\
\hline \multicolumn{2}{|c|}{$11-20$} & 25,6 & 5,7 & 3,6 & 5,5 & 13,5 & 0,0 \\
\hline \multicolumn{2}{|c|}{$21-30$} & 27,9 & 17,1 & 3,6 & 16,7 & 22,5 & 0,0 \\
\hline \multicolumn{2}{|c|}{$31-40$} & 16,0 & 14,3 & 0,0 & 11,1 & 25,7 & 40,0 \\
\hline \multicolumn{2}{|c|}{$41-50$} & 7,5 & 17,1 & 0,0 & 5,5 & 20,7 & 20,0 \\
\hline \multicolumn{2}{|c|}{$51-60$} & 2,1 & 20,0 & 0,0 & 16,7 & 9,5 & 0,0 \\
\hline \multicolumn{2}{|c|}{$61-70$} & 0,3 & 11,4 & 0,0 & 0,0 & 1,8 & 0,0 \\
\hline \multicolumn{2}{|c|}{$71-80$} & 0,3 & 8,6 & 0,0 & 11,1 & 0,9 & 0,0 \\
\hline \multicolumn{2}{|c|}{$81-90$} & 0,0 & 4,3 & 0,0 & 0,0 & 0,4 & 0,0 \\
\hline \multicolumn{2}{|c|}{$91-100$} & 0,0 & 1,4 & 0,0 & 5,5 & 0,0 & 0,0 \\
\hline \multirow{2}{*}{\multicolumn{2}{|c|}{$\begin{array}{l}\text { Distância da margem }(\mathrm{cm}) \\
\text { Externamente às margens }\end{array}$}} & 475 & 84 & 15 & 14 & 178 & 5 \\
\hline & & & & & & & \\
\hline \multicolumn{2}{|c|}{ Superior a 300} & 0,2 & 0,0 & 6,7 & 14,3 & 0,6 & 0,0 \\
\hline \multicolumn{2}{|c|}{$251-300$} & 0,0 & 0.0 & 0,0 & 0,0 & 0,0 & 0,0 \\
\hline \multicolumn{2}{|c|}{$201-250$} & 1,3 & 0,0 & 13,3 & 14,3 & 0,0 & 20,0 \\
\hline \multicolumn{2}{|c|}{$151-200$} & 0,8 & 0,0 & 20,0 & 14,3 & 0,0 & 0,0 \\
\hline \multicolumn{2}{|c|}{$101-150$} & 0,2 & 0,0 & 26,7 & 0,0 & 0,6 & 60,0 \\
\hline \multicolumn{2}{|c|}{$51-100$} & 0,4 & 0,0 & 26,7 & 14,3 & 0,0 & 20,0 \\
\hline \multicolumn{2}{|c|}{$1-50$} & 1,3 & 3,6 & 6,7 & 7,1 & 6,2 & 0,0 \\
\hline Em direção ao ce & do açude & & & & & & \\
\hline $0-5$ & & 17,4 & 22,6 & 0,0 & 28,6 & 18,0 & 0,0 \\
\hline $51-1$ & & 11,4 & 2,4 & 0,0 & 7,1 & 10,7 & 0,0 \\
\hline $101-$ & & 10,7 & 2,4 & 0,0 & 0,0 & 10,1 & 0,0 \\
\hline $151-2$ & & 14,7 & 4,8 & 0,0 & 0,0 & 7,3 & 0,0 \\
\hline $201-2$ & & 9,9 & 7,1 & 0,0 & 0,0 & 7,9 & 0,0 \\
\hline $251-3$ & & 8,2 & 8,3 & 0,0 & 0,0 & 6,7 & 0,0 \\
\hline $301-3$ & & 4,2 & 4,8 & 0,0 & 0,0 & 6,7 & 0,0 \\
\hline $351-$ & & 6.9 & 14,3 & 0,0 & 0,0 & 9,5 & 0,0 \\
\hline $401-$ & & 4,2 & 2,4 & 0,0 & 0,0 & 5,6 & 0,0 \\
\hline $451-5$ & & 4,6 & 14,3 & 0,0 & 0,0 & 5,6 & 0,0 \\
\hline $501-5$ & & 0,4 & 1,2 & 0,0 & 0,0 & 1,1 & 0,0 \\
\hline $551-$ & & 3,1 & 11,8 & 0,0 & 0,0 & 3,4 & 0,0 \\
\hline Tipo de substrato & & 518 & 81 & 32 & 15 & 189 & 6 \\
\hline Vegetaçăo herbácea & Folha & 56,6 & 59,2 & 0,0 & 0,0 & 38,1 & 0,0 \\
\hline & Talo & 33,4 & 34,6 & 0,0 & 26,7 & 36,5 & 0,0 \\
\hline & Entre folhas & 6,7 & 2,5 & 3,1 & 0,0 & 21,7 & 0,0 \\
\hline Vergataçăo arbustiva & Ramo & 0,0 & 0,0 & 3,1 & 20,0 & 0,5 & 66.7 \\
\hline & Folha & 1,0 & 3.7 & 3,1 & 33,3 & 2,6 & 0,0 \\
\hline Nympheaceae & & 2,1 & 0,0 & 0,0 & 0,0 & 0,5 & 0,0 \\
\hline Solo & & 0,2 & 0,0 & 3,1 & 0,0 & 0,0 & 16,7 \\
\hline Recob & por vegetaçăo & 0,0 & 0,0 & 87,5 & 20,0 & 0,0 & 16,7 \\
\hline Direcionamento em & ção à margem & 507 & 74 & 23 & 14 & 179 & 3 \\
\hline Voltado em direçăo op & a à margem & 19,5 & 31,1 & 26,1 & 35,7 & 34,1 & 0,0 \\
\hline Voltado para a marger & & 13,4 & 21,6 & 69,6 & 35,7 & 24,0 & 100,0 \\
\hline Paralelo à margem & & 67,1 & 47,3 & 4,3 & 28,6 & 41,9 & 0,0 \\
\hline Posição em relação & substrato & 453 & 83 & 2 & 8 & 145 & 2 \\
\hline Paralelo & & 42,4 & 54,2 & 100,0 & 62,5 & 73,1 & 0,0 \\
\hline Perpendicular & & 41,5 & 33,7 & 0,0 & 25,0 & 17,2 & 100,0 \\
\hline Diagonal & & 16,1 & 12,0 & 0,0 & 12,5 & 9,6 & 0,0 \\
\hline
\end{tabular}




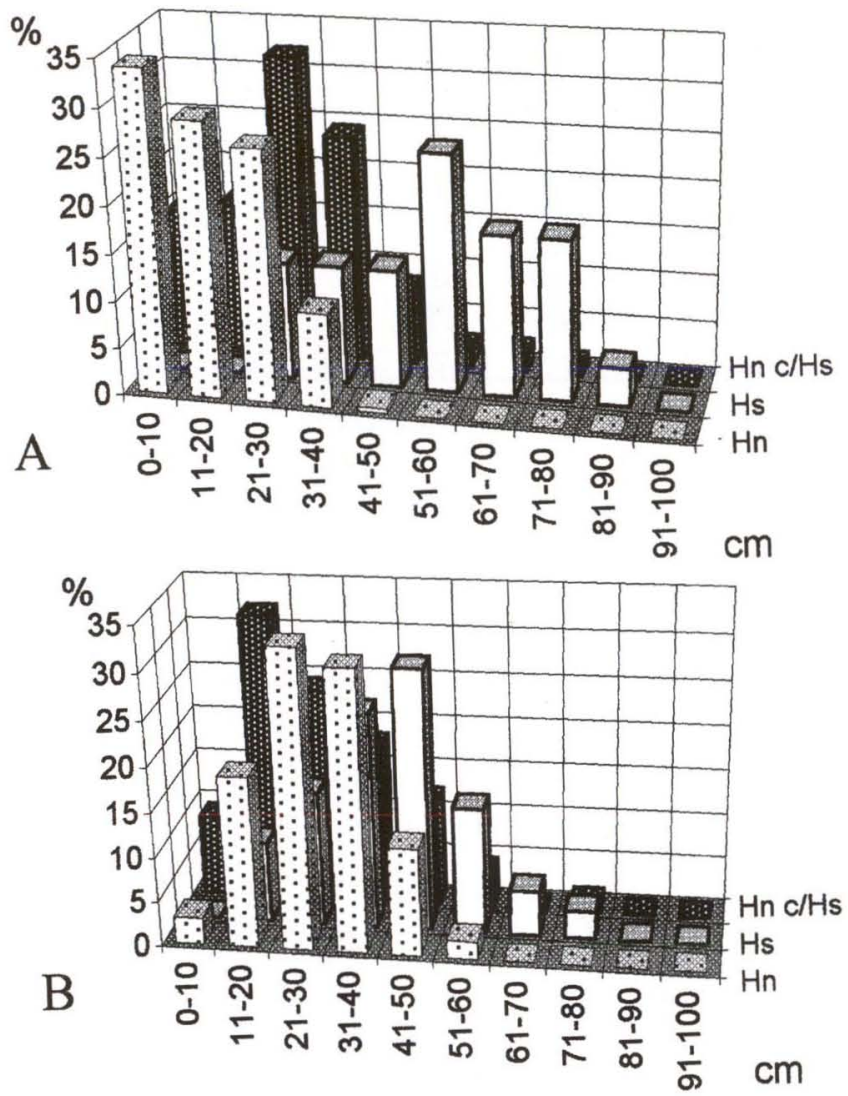

Fig. 1. Altura do sítio de vocalização dos machos de Hyla nana, antes (Hn-a) e depois (Hn-d) da chegada ao açude dos machos de H. sanborni (Hs), na estação chuvosa de 1993/1994 e de 1994/1995.

Os machos de Hyla sanborni vocalizaram em locais mais elevados que os de $H$. nana, ambos preponderantemente empoleirados na vegetação herbácea emergente (Tab. I). As duas espécies diferiram significativamente quanto às médias de altura e de distância da margem $(\mathrm{t}=12,98, \mathrm{gl}=133, \mathrm{p}<0,01 \mathrm{e} \mathrm{t}=3,50, \mathrm{gl}=579$, $\mathrm{p}<0,01$, respectivamente), mas assumiram a mesma posição durante a emissão de vocalização: paralela ou perpendicularmente à folha ou ramo, mas paralelamente à superfície da água (Tab. I). A análise sazonal da altura do sítio de vocalização evidencia que o padrão de ocupação espacial descrito acima se mantém ao longo do tempo, a despeito dos machos de $H$. nana ocuparem o açude em novembro, antes da chegada dos machos de H. sanborni, em janeiro (Fig. 1). Desde o início do seu período de ocorrência, H. sanborni estabelece seu sítio de vocalização em locais mais elevados, enquanto a altura do sítio de vocalização dos machos de $H$. nana acompanha o crescimento da vegetação do açude, que se regenera após o açude acumular água das primeiras chuvas (Fig. 2). 


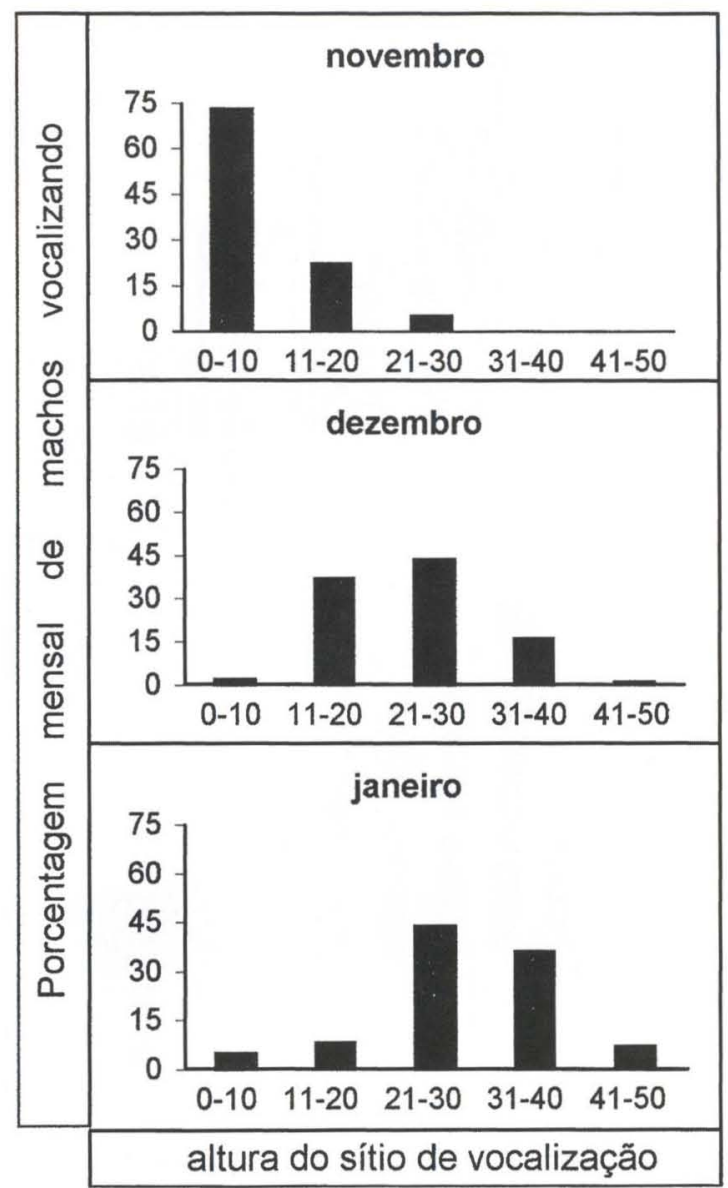

Fig. 2. Altura do sítio de vocalização dos machos de Hyla nana, conforme a vegetação do açude estava se regenerando, no período de novembro a janeiro de 1994/1995.

Machos de Scinax fuscomarginatus vocalizaram entre 3 e $110 \mathrm{~cm}$ de altura, preponderantemente sobre folhas e ramos da vegetação herbácea emergente, paralelamente à superfície da água (Tab. I). Machos de Hyla raniceps vocalizaram entre $0 \mathrm{e}$ $35 \mathrm{~cm}$ de altura, distantes da margem do açude, preponderantemente sobre ramos da vegetação arbustiva, com a cabeça voltada para o centro do açude (Tab. I).

Os machos de Physalaemus cuvieri, $P$. centralis, $P$. nattereri e $P$. fuscomaculatus vocalizaram em locais com 0 a $5 \mathrm{~cm}$ de profundidade, entre 0 e $50 \mathrm{~cm}$ da margem em direção ao centro do açude, preponderantemente em locais recobertos por vegetação herbácea (Tab. II). Não houve diferença significativa entre as médias de profundidade e de distância da margem entre os machos de cada par de espécies.

Os machos de Leptodactylus fuscus e de L. podicipinus também vocalizaram na margem do açude (Tab. II), mas L. fuscus em locais secos e L. podicipinus em 
pequenas elevações de terra acima da superfície da água, sob moitas de Poaceae e Cyperaceae. As médias de profundidade e de distância da margem, do sítio de vocalização dos machos dessas duas espécies, foi significativamente diferente $(\mathrm{t}=$ $2,60, \mathrm{gl}=58, \mathrm{p}<0,05$ e $\mathrm{t}=4,68, \mathrm{gl}=47, \mathrm{p}<0,01$, respectivamente).

Tabela II. Distribuição de freqüência ao longo das quatro variáveis do sítio de vocalização, determinadas para oito espécies de anuros que vocalizaram sobre o solo ou flutuando na água, no açude temporário em Nova Itapirema, São Paulo. (Bp) Bufo paracnemis, (Eo) Elachistocleis ovalis, (Pce) Physalaemus centralis, (Pcu) P. cuvieri, (Pfm) P. fuscomaculatus, (Pn) P. nattereri, (Pp) Pseudis paradoxa, (Lfu) Leptodactylus fuscus, (Lp) L. podicipinus, os números em negrito, na frente de cada variável, indicam o tamanho da amostra para cada espécie.

\begin{tabular}{|c|c|c|c|c|c|c|c|c|c|}
\hline Sitio de vocalização & Pce & Pcu & $\mathrm{Pfm}$ & $\mathrm{Pn}$ & Lfu & $L p$ & $\mathrm{Bp}$ & Eo & $\mathrm{Pp}$ \\
\hline Profundidade $(\mathrm{cm})$ & 42 & 45 & 12 & 17 & 38 & 46 & 14 & 7 & 6 \\
\hline $0-3$ & 92,8 & 86,7 & 100,0 & 88,2 & 100,0 & 95,6 & 35,7 & 100,0 & 0,0 \\
\hline $4-7$ & 2,4 & 13,3 & 0,0 & 11,8 & 0,0 & 4,3 & 14,3 & 0,0 & 0,0 \\
\hline $8-11$ & 4,8 & 0,0 & 0,0 & 0,0 & 0,0 & 0,0 & 21,4 & 0,0 & 0,0 \\
\hline $12-15$ & 0,0 & 0,0 & 0,0 & 0,0 & 0,0 & 0,0 & 7,1 & 0,0 & 33,3 \\
\hline $16-19$ & 0,0 & 0,0 & 0.0 & 0,0 & 0,0 & 0,0 & 0,0 & 0,0 & 0,0 \\
\hline $20-23$ & 0,0 & 0,0 & 0,0 & 0,0 & 0,0 & 0,0 & 14,3 & 0,0 & 16,7 \\
\hline $24-27$ & 0,0 & 0,0 & 0,0 & 0,0 & 0,0 & 0,0 & 7,1 & 0,0 & 50,0 \\
\hline \multicolumn{10}{|l|}{ Externamente as margens } \\
\hline Superior a 150 & 0,0 & 0,0 & 0,0 & 0,0 & 0,0 & 0,0 & 0,0 & 0,0 & 0,0 \\
\hline $101-150$ & 0,0 & 0,0 & 0,0 & 0,0 & 8,8 & 0,0 & 0,0 & 0,0 & 0,0 \\
\hline $51-100$ & 0,0 & 2,6 & 0,0 & 0,0 & 32,3 & 2,2 & 0,0 & 0,0 & 0,0 \\
\hline $1-50$ & 2,7 & 5,1 & 25,0 & 0,0 & 47,0 & 4,4 & 0,0 & 20,0 & 0,0 \\
\hline \multicolumn{10}{|l|}{ Em direção ao centro do açude } \\
\hline 0.50 & 86 & 89 & 75 & 100 & 11 & 88 & 71 & 80 & 20 \\
\hline $51-100$ & 2,7 & 2,6 & 0,0 & 0,0 & 0,0 & 0,0 & 7,1 & 0,0 & 0,0 \\
\hline $101-150$ & 2,7 & 0,0 & 0,0 & 0,0 & 0,0 & 2,2 & 7,1 & 0,0 & 20,0 \\
\hline $151-200$ & 0,0 & 0,0 & 0,0 & 0,0 & 0,0 & 0,0 & 7,1 & 0,0 & 20,0 \\
\hline $201-250$ & 0,0 & 0,0 & 0,0 & 0,0 & 0,0 & 0,0 & 0,0 & 0,0 & 20,0 \\
\hline $251-300$ & 5,4 & 0,0 & 0,0 & 0,0 & 0,0 & 0,0 & 7,1 & 0,0 & 20,0 \\
\hline Acima de 300 & 0,0 & 0,0 & 0,0 & 0,0 & 0,0 & 2,2 & 0,0 & 0,0 & 0,0 \\
\hline Tipo de substrato & 39 & 41 & 12 & 17 & 32 & 47 & 14 & 2 & 5 \\
\hline Em área com herbáceas & 66,7 & 51,2 & 83,3 & 58,8 & 68,7 & 48,9 & 42,8 & 100,0 & 0,0 \\
\hline Sob touceira de gramineas & 17,9 & 17,1 & 0,0 & 11,8 & 0,0 & 36,2 & 0,0 & 0,0 & 0,0 \\
\hline Sobre o solo nu & 7,7 & 4,9 & 8,3 & 5,9 & 28,1 & 2,1 & 28,6 & 0,0 & 0,0 \\
\hline Em depressão em solo nu & 0,0 & 9,7 & 8,3 & 0,0 & 3,1 & 0,0 & 0,0 & 0,0 & 0,0 \\
\hline Em depressão sob vegetação & 5,1 & 4,9 & 0,0 & 0,0 & 0,0 & 12,8 & 0,0 & 0,0 & 0,0 \\
\hline Em concavidade no barranco & 0,0 & 0,0 & 0,0 & 23,5 & 0,0 & 0,0 & 0,0 & 0,0 & 0,0 \\
\hline No açude, em área sem vegetaçăo & 0,0 & 0,0 & 0,0 & 0,0 & 0,0 & 0,0 & 14,3 & 0,0 & 0,0 \\
\hline No açude, em área com vegetaçăo & 0,0 & 0,0 & 0,0 & 0,0 & 0,0 & 0,0 & 14,3 & 0,0 & 0,0 \\
\hline Flutuando & 2,5 & 12,2 & 0,0 & 0,0 & 0,0 & 0,0 & 0,0 & 0,0 & 100,0 \\
\hline Direcionamento em relaçăo à margem & 39 & 34 & 4 & 13 & 30 & 33 & 17 & 2 & 4 \\
\hline Voltado em direção oposta à margem & 17,9 & 41,2 & 0,0 & 0,0 & 13,3 & 18,2 & 0,0 & 0,0 & 75,0 \\
\hline Voltado para o centro do açude & 41,0 & 47,0 & 50,0 & 61,5 & 73,3 & 42,4 & 88,2 & 50,0 & 25,0 \\
\hline Paralelo à margem & 41,0 & 11,8 & 50,0 & 38,5 & 13,3 & 39,4 & 11,8 & 50,0 & 0,0 \\
\hline
\end{tabular}

As três espécies restantes, Bufo paracnemis, Pseudis paradoxa e Elachistocleis ovalis, utilizaram áreas diferentes como sítio de vocalização (Tab. II). Apesar de $B$. paracnemis e $E$. ovalis terem vocalizado em locais com a mesma profundidade e distância da margem, ocorreram em áreas diferentes do açude: B. paracnemis na 
margem em barranco e E. ovalis na margem plana. Machos de P. paradoxa vocalizaram flutuando na água, preponderantemente em locais com profundidade superior a $20 \mathrm{~cm}$.

Houve grande sobreposição ao longo das quatro variáveis do sítio de vocalização, sendo que a distância da margem e o tipo de substrato foram mais partilhados que a altura ou a profundidade do sítio de vocalização (Tabs III, IV). A sobreposição multidimensional, considerando simultaneamente todas as variáveis do sítio de vocalização, também foi elevada: sete (47\%) das 15 combinações possíveis de pares de espécies que vocalizaram empoleiradas e $26(72 \%)$ das 36 que vocalizaram sobre o solo ou flutuando na água tiveram elevada sobreposição multidimensional ( $\mathrm{C}_{\mathrm{H}} \geq 0,55$ segundo PUTMAN 1994). No entanto, apenas $20 \%$ das combinações possíveis de pares de espécies que vocalizaram empoleiradas e $28 \%$ das que vocalizaram apoiadas sobre o solo ou flutuando apresentaram valores elevados de sobreposição para todas as variáveis do sítio de vocalização. Dos quatro pares de espécies taxonomicamente próximas, Physalaemus cuvieri e $P$. centralis, $P$. nattereri e P. fuscomaculatus, apresentaram os maiores valores de sobreposição (Tab. IV). No entanto, pares de espécies não relacionadas apresentaram o mesmo ou maior grau de sobreposição (Tabs III, IV).

Tabela III. Sobreposição de nicho, calculada pelo indice de similaridade de Morisita-Horn para as cinco variáveis do sítio de vocalização, entre as seis espécies que vocalizaram empoleiradas na vegetação, no açude de água temporária em Nova Itapirema, São Paulo. (Hn) Hyla nana, (Hr) H. raniceps, (Hs) H. sanborni, (Sfm) Scinax fuscomarginatus, (Sfu) S. fuscovarius, (Ssi) S. similis.

\begin{tabular}{|c|c|c|c|c|c|c|}
\hline \multirow[t]{2}{*}{ Pares de espécies } & \multirow[t]{2}{*}{ Altura } & \multirow[t]{2}{*}{ Distância da margem } & \multirow[t]{2}{*}{ Tipo de substrato } & \multicolumn{2}{|c|}{$\begin{array}{c}\text { Direcionamento em relaçáo à } \\
\text { superficie da(o) }\end{array}$} & \multirow[t]{2}{*}{$\begin{array}{l}\text { Sobreposição } \\
\text { multidimensional }\end{array}$} \\
\hline & & & & Água/solo & Substrato & \\
\hline $\mathrm{Hn} / \mathrm{Hs}$ & 0,57 & 0,77 & 0,990 & 0,93 & 0,97 & 0,91 \\
\hline $\mathrm{Hn} / \mathrm{Sfu}$ & 0,38 & 0,03 & 0,004 & 0,21 & 0,61 & 0,30 \\
\hline $\mathrm{Hn} / \mathrm{Ssi}$ & 0,77 & 0,46 & 0,260 & 0,71 & 0,92 & 0,67 \\
\hline $\mathrm{Hn} / \mathrm{Sfm}$ & 0,82 & 0,94 & 0,920 & 0,89 & 0,83 & 0,87 \\
\hline $\mathrm{Hn} / \mathrm{Hr}$ & 0,55 & 0,01 & 0,001 & 0,08 & 0,60 & 0,26 \\
\hline Hs/Sfu & 0,02 & 0,02 & 0,003 & 0,41 & 0,76 & 0,29 \\
\hline $\mathrm{Hs} / \mathrm{Ssi}$ & 0,63 & 0,49 & 0,290 & 0,87 & 0,98 & 0,69 \\
\hline $\mathrm{Hs} / \mathrm{Sfm}$ & 0,84 & 0,83 & 0,890 & 0,98 & 0,93 & 0,91 \\
\hline $\mathrm{Hs} / \mathrm{Hr}$ & 0,36 & 0,0 & 0,000 & 0,21 & 0,48 & 0,24 \\
\hline Sfu/Ssi & 0,55 & 0,50 & 0,370 & 0,67 & 0,85 & 0,62 \\
\hline Sfu/Sfm & 0,11 & 0,04 & 0,010 & 0,46 & 0,93 & 0,41 \\
\hline $\mathrm{Sfu} / \mathrm{Hr}$ & 0,61 & 0,74 & 0,270 & 0,82 & 0,00 & 0,41 \\
\hline Ssi/Sfm & 0,67 & 0,49 & 0,370 & 0,92 & 0,98 & 0,76 \\
\hline $\mathrm{Ssi} / \mathrm{Hr}$ & 0,66 & 0,19 & 0,440 & 0,35 & 0,34 & 0,37 \\
\hline $\mathrm{Sfm} / \mathrm{Hr}$ & 0,60 & 0,01 & 0,008 & 0,20 & 0,22 & 0,20 \\
\hline
\end{tabular}

De modo geral, a amplitude do sítio de vocalização foi grande, principalmente quanto a posição em relação a margem do açude (Tab. V). Foram consideradas generalistas as doze espécies que apresentaram nicho amplo $[\mathrm{B}>2,36$, segundo HEYER \& BELLIN (1973)] para, pelo menos, duas variáveis do sítio de vocalização (Tab. V). 
Tabela IV. Sobreposição de nicho, calculada pelo indice de similaridade de Morisita-Horn para as quatro variáveis do sítio de vocalização, entre as nove espécies que vocalizaram sobre o solo ou flutuando na água, no açude temporário em Nova Itapirema, São Paulo. (Bp) Bufo paracnemis, (Eo) Elachistocleis ovalis, (Pce) Physalaemus centralis, (Pcu) P. cuvieri, (Pfm) P. fuscomaculatus, $(\mathrm{Pn})=P$. nattereri, $(\mathrm{Pp})$ Pseudis paradoxa, $(\mathrm{Lfu})$ Leptodactylus fuscus, $(\mathrm{Lp})$ L. podicipinus.

\begin{tabular}{|c|c|c|c|c|c|}
\hline $\begin{array}{l}\text { Pares de } \\
\text { espécies }\end{array}$ & Profundidade & $\begin{array}{l}\text { Diståncia da } \\
\text { margem }\end{array}$ & $\begin{array}{l}\text { Tipo de } \\
\text { substrato }\end{array}$ & $\begin{array}{c}\text { Direcionamento } \\
\text { em relação à } \\
\text { margem }\end{array}$ & $\begin{array}{l}\text { Sobreposição } \\
\text { multidimensional }\end{array}$ \\
\hline Pcu/Pce & 0,99 & 1,00 & 0,93 & 0,75 & 0,95 \\
\hline Pcu/Pn & 1,00 & 0,99 & 0,79 & 0,69 & 0,93 \\
\hline Pcu/Pfm & 0,98 & 0,95 & 0,82 & 0,60 & 0,90 \\
\hline Pcu/Lfu & 0,98 & 0,24 & 0,80 & 0,85 & 0,73 \\
\hline Pcu/Lp & 0,99 & 1,00 & 0,89 & 0,77 & 0,95 \\
\hline Pcu/Bp & 0,67 & 0,96 & 0,21 & 0,70 & 0,71 \\
\hline PculEo & 1,00 & 0,98 & 0,66 & 0,34 & 0,77 \\
\hline $\mathrm{Pcu} / \mathrm{Pp}$ & 0,00 & 0,35 & 0,09 & 0,54 & 0,23 \\
\hline Pce/Pn & 0,99 & 0,98 & 0,82 & 0,88 & 0,95 \\
\hline Pce/Pfm & 0,99 & 0,95 & 0,89 & 0,91 & 0,95 \\
\hline Pce/Lfu & 0,99 & 0,21 & 0,91 & 0,75 & 0,75 \\
\hline Pce/Lp & 0,99 & 1,00 & 0,91 & 0,98 & 0,98 \\
\hline Pce/Bp & 0,64 & 0,98 & 0,24 & 0,63 & 0,68 \\
\hline Pce/Eo & 0,98 & 0,97 & 0,80 & 0,20 & 0,76 \\
\hline $\mathrm{Pce} / \mathrm{Pp}$ & 0,00 & 0,40 & 0,04 & 0,39 & 0,19 \\
\hline $\mathrm{Pn} / \mathrm{Pfm}$ & 0,98 & 0,92 & 0,82 & 0,97 & 0,94 \\
\hline $\mathrm{Pn} / \mathrm{Lfu}$ & 0,98 & 0,18 & 0,71 & 0,86 & 0,68 \\
\hline $\mathrm{Pn} / \mathrm{Lp}$ & 0,99 & 0,99 & 0,71 & 0,92 & 0,95 \\
\hline $\mathrm{Pn} / \mathrm{Bp}$ & 0,66 & 0,93 & 0,42 & 0,86 & 0,77 \\
\hline Pn/Eo & 1,00 & 0,95 & 0,56 & 0,00 & 0,66 \\
\hline $\mathrm{Pn} / \mathrm{Pp}$ & 0,00 & 0,33 & 0,00 & 0,16 & 0,13 \\
\hline Pfm/Lfu & 1,00 & 0,43 & 0,86 & 0,73 & 0,81 \\
\hline $\mathrm{Pfm} / \mathrm{Lp}$ & 1,00 & 0,95 & 0,67 & 0,93 & 0,93 \\
\hline $\mathrm{Pfm} / \mathrm{Bp}$ & 0,59 & 0,93 & 0,49 & 0,72 & 0,70 \\
\hline Pfm/Eo & 0,98 & 1,00 & 0,73 & 0,00 & 0,69 \\
\hline $\mathrm{Pfm} / \mathrm{Pp}$ & 0,00 & 0,36 & 0,00 & 0,12 & 0,10 \\
\hline Lfu/Lp & 1,00 & 0,23 & 0,71 & 0,76 & 0,72 \\
\hline Lfu/Bp & 0,59 & 0,19 & 0,32 & 0,94 & 0,51 \\
\hline Lfu/Eo & 0,98 & 0,37 & 0,82 & 0,05 & 0,60 \\
\hline Lfu/Pp & 0,00 & 0,09 & 0,00 & 0,28 & 0,07 \\
\hline Lp/Bp & 0,62 & 0,96 & 0,07 & 0,68 & 0,65 \\
\hline Lp/Eo & 0,99 & 0,97 & 0,67 & 0,24 & 0,75 \\
\hline$L p / P p$ & 0,00 & 0,36 & 0,00 & 0,40 & 0,17 \\
\hline $\mathrm{Bp} / \mathrm{Eo}$ & 0,67 & 0,94 & 0,00 & 0,00 & 0,37 \\
\hline $\mathrm{Bp} / \mathrm{Pp}$ & 0,27 & 0,51 & 0,00 & 0,20 & 0,22 \\
\hline Eo/Pp & 0,00 & 0,36 & 0,00 & 0,92 & 0,34 \\
\hline
\end{tabular}

A análise de similaridade revelou, para os anuros que vocalizaram empoleirados, a existência de dois agrupamentos: (1) formado por Hyla nana, H. sanborni, Scinax fuscomarginatus e $S$. similis, que vocalizaram empoleiradas na vegetação herbácea ou arbustiva; (2) formado por Scinax fuscovarius e Hyla raniceps, que vocalizaram sobre o solo (Fig. 3). Entre os anuros que vocalizaram apoiados sobre o solo ou flutuando na água, a análise de similaridade também revelou a existência de dois agrupamentos de espécies: (1) formado pelas quatro espécies de Physalaemus e por Leptodactylus podicipinus, cujos machos vocalizaram preponderantemente na margem do açude, em locais alagados recobertos por vegetação herbácea, 


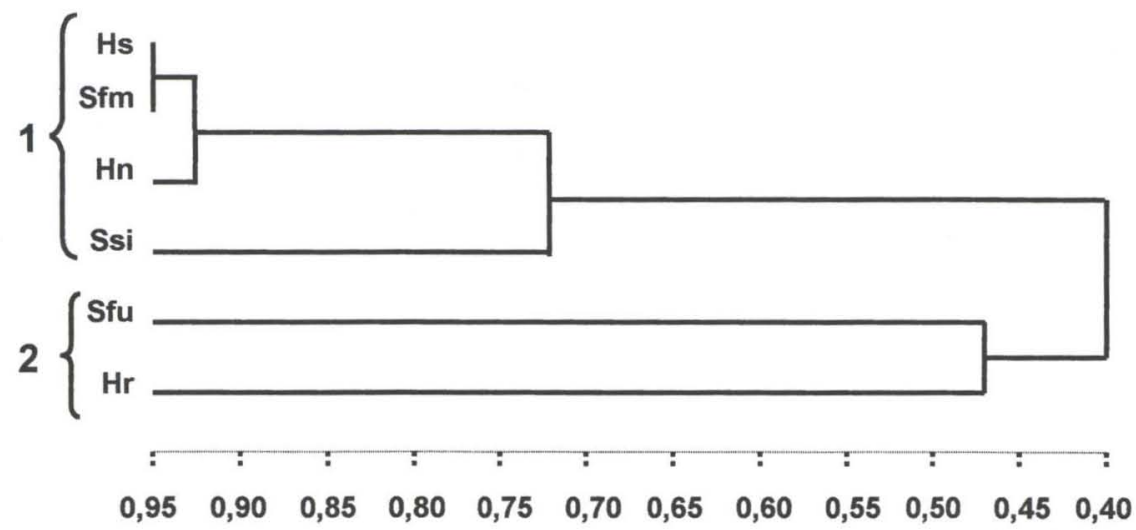

Fig. 3. Similaridade do sítio de vocalização entre as seis espécies de anuros que vocalizaram empoleirados na vegetação, no açude temporário em Nova Itapirema, São Paulo. (Hn) Hyla nana, (Hs) H. sanborni, (Hr) H. raniceps, (Sfu) Scinax fuscovarius, (Ssi) S. similis, (Sfm) $S$. fuscomarginatus.

Tabela V. Amplitude de nicho para quatro variáveis do sítio de vocalização das 15 espécies de anuros encontradas no açude temporário em Nova Itapirema, São Paulo.

\begin{tabular}{lcccc}
\hline \multicolumn{1}{c}{ Espécies } & Profundidade/Altura & $\begin{array}{c}\text { Distância da } \\
\text { margem }\end{array}$ & $\begin{array}{c}\text { Tipo de } \\
\text { substrato }\end{array}$ & $\begin{array}{c}\text { Direcionamento em } \\
\text { relaçăo à margem }\end{array}$ \\
\hline Bufo paracnemis & 6,53 & 1,88 & 3,27 & 2,47 \\
Elachistocleis ovalis & 1,32 & 1,47 & 1,00 & 2,00 \\
Hyla nana & 1,71 & 9,76 & 2,29 & 2,06 \\
Hyla samborni & 6,90 & 7,95 & 2,12 & 3,25 \\
Hyla raniceps & 2,78 & 2,27 & 2,00 & 1,00 \\
Leptodactylus fuscus & 1,00 & 2,88 & 2,27 & 3,26 \\
Leptodactylus podicipinus & 1,61 & 1,26 & 2,99 & 3,29 \\
Physalaemus centralis & 2,23 & 1,33 & 2,94 & 4,62 \\
Physalaemus cuvieri & 2,50 & 1,24 & 4,26 & 2,67 \\
Physalaemus fuscomaculatus & 1,95 & 1,60 & 2,67 & 2,97 \\
Physalaemus nattereri & 2,47 & 1,00 & 3,96 & 1,60 \\
Pseudis paradoxa & 4,50 & 5,00 & 1,00 & 3,79 \\
Scinax fuscomarginatus & 5,27 & 10,51 & 3,07 & 2,66 \\
Scinax fuscovarius & 1,16 & 4,79 & 1,30 & 4,67 \\
Scinax similis & 6,00 & 5,81 & 3,82 & \\
\hline
\end{tabular}

com 0 a $3 \mathrm{~cm}$ de profundidade e 0 a $50 \mathrm{~cm}$ de distância da margem; (2) formado por três espécies pertencentes a famílias diferentes, cujos machos vocalizaram voltados para o centro do açude: Leptodactylus fuscus e Elachistocleis ovalis em áreas secas na margem do açude e Bufo paracnemis no interior do açude, preponderantemente em locais com profundidade da coluna d'água entre 4 e $27 \mathrm{~cm}$ (Fig. 4).

A estrutura física do canto de anúncio dos machos dos pares de espécies taxonomicamente próximas diferiu quanto a (I) freqüência de emissão: entre 3,0 e $4,8 \mathrm{kHz}$ para Hyla nana e entre 4,6 e $6,6 \mathrm{kHz}$ para $H$. sanborni e (II) duração das notas: mais longas em Scinax fuscovarius (0,36 s), Physalaemus centralis $(0,54 \mathrm{~s})$ e $P$. fuscomaculatus $(0,51 \mathrm{~s})$ que nos respectivos pares congenéricos, $S$. similis $(0,18$ s), P. cuvieri ( $0,28 \mathrm{~s})$ e $P$. nattereri $(0,05 \mathrm{~s})$ (Figs 5-6). 


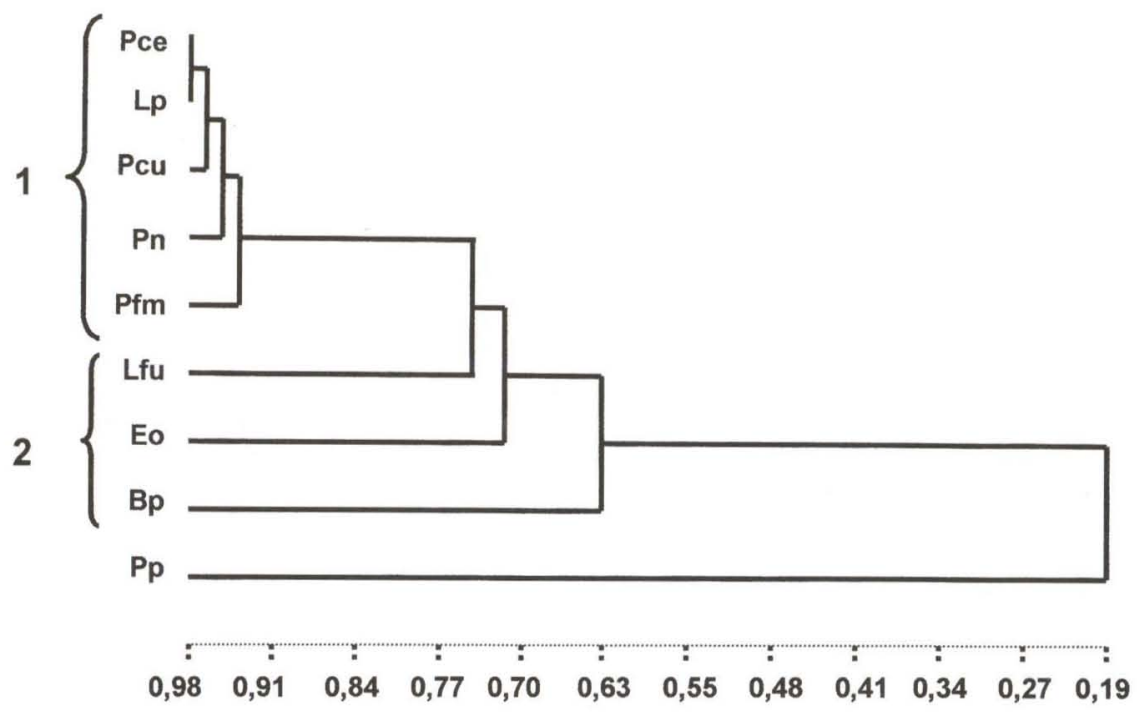

Fig. 4. Similaridade do sítio de vocalização entre as nove espécies de anuros que vocalizaram apoiados sobre o solo ou flutuando na água, no açude temporário em Nova Itapirema, São Paulo. (Pcu) Physalaemus cuvieri, (Pce) P. centralis, (Pn) P. nattereri, (Pfm) P. fuscomaculatus, (Lfu) Leptodactylus fuscus, (Lp) L. podicipinus, (Bp) Bufo paracnemis, (Eo) Elachistocleis ovalis, (Pp) Pseudis paradoxa.

De modo geral, a distância até o vizinho mais próximo, entre machos da mesma espécie, foi maior que entre machos de espécies diferentes (Tab. VI). As exceções foram Scinax fuscovarius e Physalaemus centralis, cujos machos mostraram tendência à agregação, com distância intraespecífica até o vizinho mais próximo menor que a distância interespecifica (Tab. VI).

Tabela VI. Distância $(\mathrm{cm})$ intraespecífica entre machos vizinhos mais próximos, e distância interespecífica entre os machos de oito espécies de anuros que vocalizaram apoiados sobre o solo e de quatro espécies de anuros que vocalizaram empoleirados na vegetação.

\begin{tabular}{|c|c|c|c|c|}
\hline Espécies & $x$ & DP & $\mathrm{CV}(\%)$ & $n$ \\
\hline \multicolumn{5}{|c|}{ Machos que vocalizaram apoiados sobre o solo } \\
\hline Bufo paracnemis & 207.1 & 117.0 & 56,5 & 7 \\
\hline Leptodactylus fuscus & 268,3 & 133,8 & 49,9 & 15 \\
\hline Leptodactylus podicipinus & 158,0 & 153,8 & 97,3 & 29 \\
\hline Physalaemus centralis & 130,1 & 145,3 & 111,7 & 32 \\
\hline Physalaemus cuvieri & 145,1 & 174,1 & 120,0 & 34 \\
\hline Physalaemus fuscomaculatus & 244,0 & 87,6 & 35,9 & 5 \\
\hline Physalaemus nattereri & 283,3 & 175,1 & 61,8 & 6 \\
\hline Scinax fuscovarius & 121,6 & 170,6 & 140,3 & 19 \\
\hline Distância interespecifica & 141,6 & 221,8 & 156,6 & 31 \\
\hline \multicolumn{5}{|c|}{ Machos que vocalizaram empoleirados } \\
\hline Hyla nana & 145,1 & 111,3 & 78,3 & 266 \\
\hline Hyla sanborni & 194,2 & 165,9 & 85,4 & 33 \\
\hline Scinax fuscomarginatus & 162,5 & 115,8 & 71,3 & 64 \\
\hline Scinax similis & 483,3 & 76.4 & 15,8 & 3 \\
\hline Diståncia interespecifica & 144,7 & 115,3 & 79,7 & 130 \\
\hline
\end{tabular}




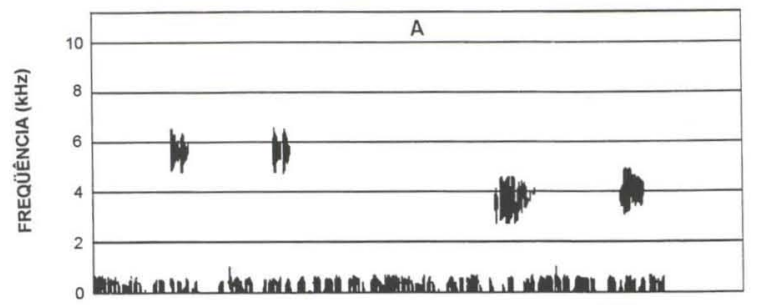

TEMPO
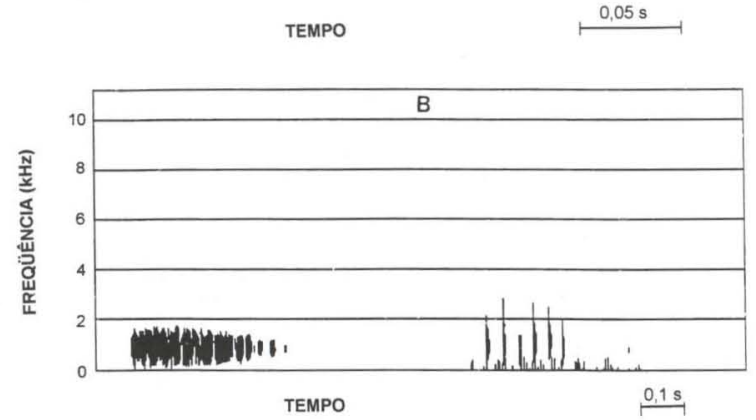

Fig. 5. Sonograma de duas notas do canto de anúncio de, da esquerda para a direita, (A) Hyla sanborni e H. nana e (B) $S$. fuscovarius e $S$. similis, no açude temporário em Nova Itapirema, São Paulo. Temperatura do ar: (A) $27^{\circ} \mathrm{C}$ e (B) $23,5^{\circ} \mathrm{C}$, filtro de 64 pontos. Os registros no sonograma abaixo de $1 \mathrm{kHz}$ correspondem a ruídos de fundo.
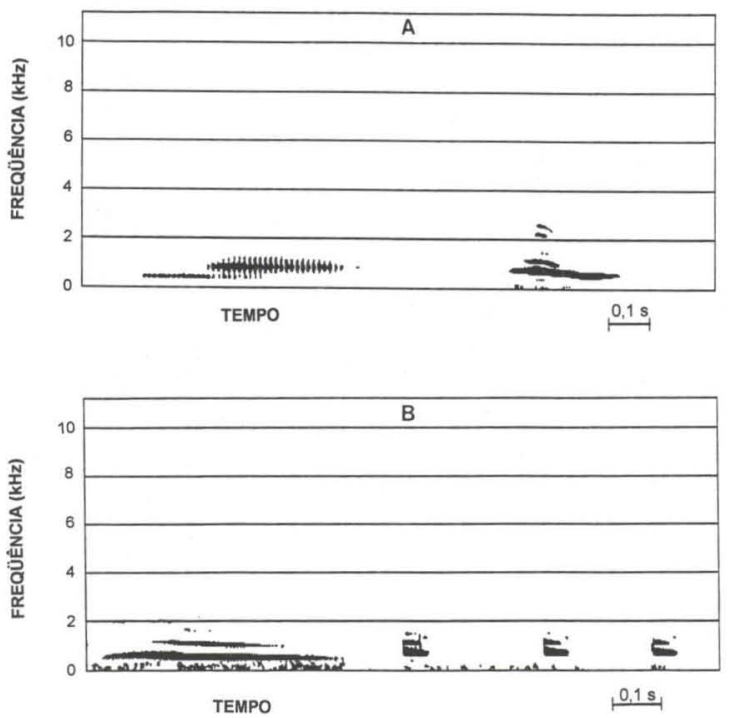

Fig. 6. Sonograma de duas notas do canto de anúncio de, da esquerda para a direita, (A) Physalaemus centralis e $P$. cuvieri e (B) $P$. fuscomaculatus e $P$. nattereri, no açude de água temporária em Nova Itapirema, São Paulo. Temperatura do ar: (A) $23,5^{\circ} \mathrm{C}$ e (B) $25,5^{\circ} \mathrm{C}$, filtro de 256 pontos. Os registros abaixo de $0,5 \mathrm{kHz}$ correspondem a ruídos de fundo. 


\section{DISCUSSÃO}

\section{Comunidade do açude}

O sítio de vocalização das espécies estudadas foi, de modo geral, idêntico ao registrado para as mesmas espécies em outras localidades (BOKERMANN 1962; BARRIO 1962, 1967; CEI 1980; MARTINS 1988; CARdoso et al. 1989; HADDAD \& SAZIMA 1992; BARRETO \& ANDRADE 1995), o que sugere que essa, dentro da amplitude de nicho de cada espécie, é uma característica conservativa. Segundo ETGES (1987) a seleção do sítio de vocalização parece ser uma extensão fillogenética adicional ao comportamento de isolamento acústico em muitas espécies de anuros.

A sobreposição do sítio de vocalização foi alta entre os anuros que vocalizaram empoleirados. No entanto, grande parte dessa sobreposição ocorreu entre extremos das curvas de distribuição, principalmente nas variáveis altura e distância da margem. A análise de agrupamento mostra que três, das seis espécies cujos machos vocalizaram empoleirados, apresentaram segregação quanto ao sítio de vocalização. É interessante que, para as variáveis ecológicas do sítio de vocalização, que envolvem características ambientais como distância da margem, altura e tipo de substrato, a sobreposição parece decorrente mais do tamanho corporal que de relações de parentesco. Assim, para estas variáveis, Scinax fuscomarginatus apresentou valores elevados de similaridade e de sobreposição de nicho com Hyla nana e H. sanborni. Já, para as variáveis de cunho comportamental, como posição no substrato e em relação à superfície da água, Scinax fuscomarginatus apresentou maior sobreposição com a congenérica $S$. similis. A pequena segregação na altura do sítio de vocalização entre os anuros que vocalizaram empoleirados pode ser explicada em função da estrutura fisionômica da vegetação do açude estudado, composta preponderantemente por vegetação herbácea, que possibilita poucas oportunidades de partilha vertical.

A sobreposição do sítio de vocalização entre os anuros que vocalizaram apoiados sobre o solo ou flutuando na água foi muito alta; poucas combinações de espécies apresentaram valores de sobreposição menores que $\mathrm{C}_{\mathrm{H}}=0,55$. A análise de agrupamento mostra grande similaridade entre as espécies; apenas Pseudis paradoxa utilizou locais diferentes como sítio de vocalização. Essa grande sobreposição do sítio de vocalização contraria os resultados obtidos por HEYER et al. (1990) e HADDAD \& SAZIMA (1992), que registraram segregação espacial em comunidades de região tropical. No entanto, resultados semelhantes foram obtidos por CARDoso et al. (1989) em duas localidades com vegetação de pequeno porte, nas quais o número de microhábitats disponíveis foi menor que o número de espécies. Já em uma localidade de mata, com maior heterogeneidade ambiental, os autores observaram que o número de microhábitats foi semelhante ao número de espécies.

Segundo CARDOSO et al. (1989), a heterogeneidade espacial é importante na determinação do número de espécies que podem explorar um dado ambiente. No presente estudo, apesar da pequena partilha ao longo da variável altura do sítio de vocalização, foi o uso do espaço tridimensional que possibilitou a partilha ao longo das dimensões tipo de substrato e distância da margem, já que as espécies de hábito 
trepador vocalizaram no interior do açude, sobre diversas espécies de plantas emergentes. Assim, o estrato vertical cria um espaço tridimensional para o estabelecimento de microhábitats e, segundo MESERVE (1977), o uso de áreas de vida tridimensionais proporciona um meio de reduzir a competição entre espécies congenéricas. Já para os anuros que vocalizaram apoiados sobre o solo ou flutuando na água, a pequena heterogeneidade ambiental pode ter limitado a diferenciação ou essas espécies podem não ter sofrido pressão para diferenciação do sítio de vocalização. Apenas duas espécies (Pseudis paradoxa e Bufo paracnemis) vocalizaram no interior do açude e apenas Leptodactylus fuscus vocalizou externamente às margens. Todas as demais espécies vocalizaram nas margens do açude. $O$ fato que a maioria das espécies registradas no açude temporário vocalizou sobre o solo, sugere que a segregação espacial não foi um fator limitante para o sucesso reprodutivo dessas espécies e, além disso, evidencia que a partilha de recursos só pode ser compreendida quando se considera as diversas variáveis envolvidas, já que essas espécies apresentaram sobreposição espacial, mas partilharam o espaço acústico.

Os dados descritivos obtidos neste estudo não permitem inferências sobre interações interespecíficas. No entanto, considerando os anuros que vocalizaram apoiados sobre o solo, apesar da alta sobreposição e da proximidade intra e interespecífica entre os machos, nenhuma interação agonística foi observada. Apenas girinos de $P$. centralis não foram abundantes e ocorreram em apenas dois dos cinco anos de estudo, o que indica que as demais espécies obtiveram sucesso reprodutivo.

A maioria das espécies foi generalista, apresentando grande amplitude de nicho. LEVINS (1968) argumenta que nicho amplo é característico de espécies adaptadas a hábitats instáveis e imprevisíveis, situação em que se enquadra o açude temporário estudado, sujeito a fortes variações no mês de início da estação chuvosa e na duração e volume de água acumulada. Assim, a grande amplitude verificada para o sítio de vocalização parece ser uma adaptação necessária à sobrevivência nesse hábitat, gerando, como conseqüência, a alta sobreposição espacial entre as espécies.

\section{Pares de espécies taxonomicamente próximas}

Os hilídeos dos dois pares de espécies taxonomicamente próximas utilizaram sítios de vocalização diferentes. Machos de Scinax fuscovarius vocalizaram sobre o solo, onde apenas alguns machos de S. similis foram observados. A altura do sítio de vocalização foi a única separação entre essas duas espécies, pois os machos ocorreram nas mesmas áreas do açude. Este fato é conseqüência de uma coincidência na distribuição espacial da vegetação arbustiva, encontrada apenas nas margens em barranco, onde machos de ambas as espécies vocalizaram: os de S. similis empoleirados e os de S. fuscovarius sobre o solo.

Machos de Hyla nana e de H. sanborni também ocorreram nas mesmas áreas do açude, mas separados pela altura do sítio de vocalização e pela diferença na freqüência dominante do canto de anúncio. Resultados semelhantes foram registrados por M.L. del Grande (comunicação pessoal) durante estudo sobre a biologia reprodutiva dessas duas espécies em Corumbataí, São Paulo. No entanto, a segre- 
gação espacial e acústica entre $H$. nana e $H$. sanborni foi maior no presente estudo, pois os machos de $H$. sanborni vocalizaram em locais mais altos ( $\mathrm{X}=47,8 \mathrm{~cm}$ no presente estudo e $\mathrm{X}=29,02 \mathrm{~cm}$ em Corumbataí) e não houve sobreposição na freqüência do canto de anúncio das duas espécies. Aparentemente essa segregação não é resultante de competição no presente, pois machos de $H$. nana vocalizaram em locais mais baixos desde antes da chegada dos machos de H. sanborni.

Os sítios de vocalização dos pares Physalaemus cuvieri/P. centralis e $P$. nattereri/P. fuscomaculatus foram iguais, mas a estrutura física do canto de anúncio foi diferente. Situação semelhante foi registrada por CARDOSO \& VIELLIARD (1990) para Hyla granosa e $H$. microderma, enquanto partilha espacial foi registrada para leptodactilídeos por DiAZ \& VALENCIA (1985) e HEYER et al. (1990), demonstrando que esse padrão de partilha não é característica relacionada ao táxon de família. Houve tendência dos machos de $P$. nattereri vocalizarem com a região posterior do corpo encostada em regiões mais elevadas do solo, como pequenos montes de terra ou margens em barranco (observação do primeiro autor). Isto separou, em parte, os machos de P. nattereri dos de P. fuscomaculatus.

Leptodactylus fuscus e L. podicipinus pertencem a diferentes grupos de espécies deste gênero e o sítio de vocalização dos machos foi diferente quanto ao tipo de substrato e a localização em relação à margem do açude.

ETGES (1987) sugere que a similaridade genética entre as espécies pode ser inversamente correlacionada com uma medida global de similaridade ecológica dos sítios de vocalização. A sobreposição de nicho entre os machos dos quatro pares de espécies taxonomicamente próximas foi alta, mas algumas espécies não congenéricas (e.g., H. sanborni e S. fuscomarginatus; P. centralis e L. podicipinus) apresentaram sobreposição igual ou maior. Esses dados mostram que a similaridade no sítio de vocalização não pode ser explicada unicamente com base na proximidade taxonômica das espécies. Isso não significa que a proximidade taxonômica não foi importante na comunidade estudada, mas, sim, que outros fatores como tamanho corporal e heterogeneidade espacial influenciaram o padrão de utilização de sítios de vocalização.

Considerando o nicho multidimensional (espaço físico e acústico), os resultados obtidos confirmam a sugestão de HEYER et al. (1990): os quatro pares de espécies próximas, apesar de ocorrerem no mesmo açude, ao mesmo tempo, apresentaram segregação ao longo de algumas dimensões do sítio de vocalização (espécies que vocalizaram empoleiradas) ou partilharam o espaço acústico (espécies que vocalizaram sobre o solo ou flutuando na água).

AGRADECIMENTOS. Os autores agradecem ao Prof. Dr. Célio F.B. Haddad, da UNESP, Campus de Rio Claro, pela confecção dos sonogramas e permissão para o uso do equipamento financiado pela Fundação de Amparo à Pesquisa do Estado de São Paulo (FAPESP), e ao Prof. Dr. Francisco Langeani pela correção do Abstract. Geisa Amadio, Geise V. de Melo, Itamar A. Martins, Mara A. Marçal, Marcelo Menin, Rodrigo P. Ribeiro, Sérgio H.S. Lins Costa e Thiago J. Izzo, estagiários em diferentes épocas, foram imprescindiveis nos trabalhos de campo. 


\section{REFERÊNCIAS BIBLIOGRÁFICAS}

ARID, F.M. \& S.F. BARCHA. 1973. Água subterrânea na formação Bauru - Região norte-ocidental do Estado de São Paulo. Bol. Ciências, São José do Rio Preto, (1): 70-101.

Barreto, L. \& G.V. AndRAde. 1995. Aspects of the reproductive biology of Physalaemus cuvieri (Anura: Leptodactylidae) in northeastern Brazil. Amphibia-Reptilia, Leiden, 16: 67-76.

Barrio, A. 1962. Los Hylidae de Punta Lara, Provincia de Buenos Aires: observaciones sistemáticas, ecológicas, y análisis espectrográfico del canto. Physis 23 (65): 129-141.

1967. Sobre la validez de Hyla sanborni K. P. Schmidt e H. uruguaya K. P. Schmidt (Anura, Hylidae). Physis 26 (73): 521-524.

Bokermann, W.C.A. 1962. Observações biológicas sobre Physalaemus cuvieri Frtz., 1826 (Amphibia, Salientia). Rev. Brasil. Biol. 22 (4): 391-399.

BOWKER, R.G. \& M.H. BowKER. 1979. Abundance and distribution of anurans in Kenian pond. Copeia 1979 (2): 278-285.

Cardoso, A.J.; G.V. Andrade \& C.F.B. Haddad. 1989. Distribuição espacial em comunidades de anfíbios (Anura) no sudeste do Brasil. Rev. Brasil. Biol. 49 (1): 241-249.

CARdoso, A.J. \& J. Vielliard. 1990. Vocalizações de anfíbios anuros de um ambiente aberto, em Cruzeiro do Sul, Estado do Acre. Rev. Brasil. Biol. 50 (1): 229-242.

CEI, J.M. 1980. Amphibians of Argentina. Monit. Zool. Ital. (N.S.). Monografia 2: 1-609.

CREUSERE, F.M. \& W.G. WhitTFORD. 1976. Ecological relationships in a desert anuran community. Herpetologica 32 (1): 7-18.

DiAZ, N.F. \& J. VAlencia. 1985. Microhabitat utilization by two leptodactylid frogs in the Andes of Central Chile. Oecologia 66 (3): 353-357.

Duellman, W.E. 1967. Courtship isolating mechanisms in Costa Rica hylid frogs. Herpetologica 23: 169-183.

Etges, W.J. 1987. Call site choice in male anurans. Copeia 1987 (4): 910-923.

Haddad, C.F.B. \& I. SaZima. 1992. Anfíbios anuros da Serra do Japi, p. 188-211. In: L.P.C. Morellato

(Ed.). História Natural da Serra do Japi: Ecologia e Preservação de uma Área Florestal no Sudeste do Brasil. Campinas, Edunicamp/Fapesp, 321p.

HeYer, W.R. \& M.S. Bellin. 1973. Ecological notes on five sympatric Leptodactylus (Amphibia, Leptodactylidae) from Ecuador. Herpetologica 29 (1): 66-72.

Heyer, W.R.; A.S. Rand; C.A.G. DA Cruz; O.L. Peixoto \& C.E. Nelson. 1990. Frogs of Boracéia. Arq. Zool., São Paulo, 31 (4): 231-410.

KREBS, C.J. 1989. Ecological Methodology. New York, Harper \& Row, 652p.

LEvins, R. 1968. Evolution in changing environments, some theoretical explorations. Monogr. Pop.

Biol. 2: 1-120.

MARTINs, M. 1988. Biologia reprodutiva de Leptodactylus fuscus em Boa Vista, Roraima (Amphibia: Anura). Rev. Brasil. Biol. 48 (4): 969-977.

MESERVE, P.L. 1977. Three-dimensional home ranges of Cricetid rodents. Jour. Mammal. 58: 549-558.

OLDHAM, R.S. \& H.C. GERHARDT. 1975. Behavioral isolating mechanisms of the treefrogs Hyla cinerea and H. gratiosa. Copeia 1975 (2): 223-231.

Putman, R.J. 1994. Community Ecology. London, Chapman \& Hall, 178p.

VIEIRA, S. 1985. Introdução à Bioestatística. Rio de Janeiro, Campus, 294p.

Recebido em 15.VI.2000; aceito em 18.V.2001 